\title{
Les fantômes de la pharmacie passée, présente et future
}

\author{
par Neil J MacKinnon
}

"Je vivrai dans le passé, le présent et le futur! " répéta Scrooge en sautant du lit. "Les leçons des trois esprits demeureront gravées dans ma mémoire. Ô Jacob Marley! que le ciel et la fête de Noël soient bénis de leurs bienfaits!" — Un conte de Noël, Charles Dickens, 1843

Cos ela peut vous sembler étrange de faire référence à un conte de Noël à ce temps-ci de l'année, mais c'est une de mes histoires préférées. J'adore la transformation de ce personnage d'avare qu'est Scrooge après qu'il a reçu la visite des trois esprits incarnant les Noëls passé, présent et futur. Les fantômes lui rappellent ses origines, lui présentent un point de vue plus global de sa situation actuelle et lui laissent entrevoir l'avenir, si jamais il décidait de continuer sur le présent chemin. S’il y avait des fantômes de la pharmacie hospitalière passée, présente et future, je me demande ce qu'ils auraient à nous dire, et si leurs révélations modifieraient nos actions présentes.

J'ai lu récemment un manuscrit inédit, rédigé par Jessie I. MacKnight, au sujet de l'exercice de la pharmacie hospitalière dans les provinces atlantiques canadiennes de 1940 à 1964. Le premier cours de pharmacie hospitalière a été offert par le Maritime College of Pharmacy (maintenant le Collège de pharmacie au sein de l'Université Dalhousie) en 1931. Dans les années 1940, les principaux enjeux de la pharmacie hospitalière au Canada étaient décrits comme «le besoin d'avoir un programme commun de pharmacie hospitalière dans les collèges de pharmacie au Canada, le type et la durée de l'apprentissage des pharmaciens d'hôpitaux, et l'édition d'un formulaire hospitalier qui fait cruellement défaut pour le Canada.» Ça vous dit quelque chose?

Aujourd'hui, nous, les pharmaciens d'hôpitaux, pouvons être fiers de nos réalisations récentes, mais nous devons quand même continuer d'aspirer à l'avancement de la profession et à un système de distribution des médicaments plus sûr et plus efficace. Il s'est écoulé une génération depuis l'arrivée des soins pharmaceutiques et, pourtant, ils ne sont pas encore systématiquement prodigués aux patients hospitalisés au Canada. Les pharmaciens sont peut-être sortis des sous-sols des hôpitaux de notre pays, mais avons-nous réussi à devenir des membres indispensables de l'équipe des soins de santé dans les unités de soins?

Alors, que pouvons-nous dire au sujet de l'avenir de la pratique de la pharmacie hospitalière? Cela est certainement sous la loupe du Conseil de la SCPH. Nous visons l'excellence en pratique avec le projet SCPH 2015, nous sommes en train de finaliser un nouveau plan stratégique, et nous avons mis sur pied un nouveau groupe de travail chargé d'examiner le rôle des techniciens en pharmacie dans notre organisation. Si vous êtes un pharmacien clinicien, je vous invite à vous imaginer ce à quoi pourrait ressembler votre propre domaine de pratique dans cinq ans, et si vous êtes un chef ou un gestionnaire de pharmacie, à vous imaginer à quoi pourrait ressembler votre service dans ce même espace de temps.

Alors, n'oublions pas notre passé, vivons dans le présent, et regardons vers le futur, en sachant tirer parti de notre riche histoire, en étant conscients de notre situation actuelle et en rêvant de ce que le futur pourrait être.

[Traduction par l'éditeur]

Neil J MacKinnon, B. Sc. (Pharm.), M. Sc. (Pharm.), Ph. D., FCSHP, est président et agent de liaison pour la vision de la SCPH. 